\title{
Evaluación de la Madurez de la Función Mantenimiento para Implementar Innovaciones en su Gestión
}

\author{
Fernando F. Espinosa y Gonzalo E. Salinas \\ Universidad de Talca, Facultad de Ingeniería, 2 Norte 685, Talca-Chile \\ (e-mail: fespinos@utalca.cl gsalinas@utalca.cl)
}

Recibido Jul. 29, 2009; Aceptado Sept. 04, 2009; Versión Final recibida Sept. 29, 2009

\begin{abstract}
Resumen
Se presenta un modelo validado e implementado en Excel, para evaluar el estado de la madurez de una organización. Esto es que la organización está perfectamente condicionada para tratar sus proyectos, basado en los aspectos de compromiso con el cambio, cultura de la organización, comunicación intra y extra departamento, uso de herramientas para gestión de proyectos y solución de los conflictos. Los resultados son entregados en forma gráfico-narrativa los cuales son un punto de partida para implementar estrategias para realizar innovaciones en el proceso de gestión del mantenimiento. Uno de los puntos principales de la metodología es la aplicación de un cuestionario, elaborado bajo el procedimiento usado en la metodología MCDA, orientado a caracterizar los aspectos nombrados en la forma de variables cuantificables, o sea, asimilar un valor o un concepto bien definido para cada estado en que las variables puedan presentarse.
\end{abstract}

Palabras clave: gestión del mantenimiento, madurez, cultura organizacional, innovación

\section{Maturity Evaluation of the Maintenance Function to Implement Innovations in its Management}

\begin{abstract}
In this paper is presented a validated and implemented model in Excel, to evaluate the state of maturity, understanding for that the organization is well conditioned to deal with their projects, based on aspects of commitment to change, organizational culture, communication inside and outside the department, use of tools for project management and conflict resolution. The results are given in a graphic-narrative form which is a starting point to implement strategies for innovation in maintenance management process. One of the principal points of the methodology is the use of a questionnaire, elaborated under the MCDA procedure, designed to characterize the aspects named in the form of quantifiable variables, that is, assimilate a value or a well-defined concept for each state in which the variables that modeling the system under study may arise.
\end{abstract}

Keyword: maintenance management, maturity, organizational culture, innovation 


\section{INTRODUCCIÓN}

Uno de los factores que influyen de forma negativa en el momento de implementar una nueva concepción o tipo de gestión del mantenimiento es el desconocimiento, o no tener una percepción completa del ambiente tecnológico y humano que está presente en la organización. Se hace necesario tener un mecanismo para tener una retroalimentación entre el estado actual de la madurez, los objetivos estratégicos y los planes de acción para mejorar la madurez de la organización (Abrunhosa y Moura e Sá, 2008; Xirogiannis y Glykas, 2007; Garengo et al., 2007).

Los aspectos humanos y tecnológicos que influyen en el proceso de implementación de la concepción del mantenimiento y de la definición del modelo de gestión, precisan ser caracterizados en la forma de variables cuantificables, o sea, asimilar un valor o un concepto bien definido para cada estado en que las variables que modelan el sistema bajo estudio puedan presentarse, el cual es el objetivo principal de este trabajo. Esta forma de análisis permite al administrador conocer el estado actual de los recursos físicos y humanos de la organización y, junto al estado final que es deseado, proponer alternativas de acción para eliminar todos los aspectos que no contribuyen de forma positiva para alcanzar el objetivo propuesto (Maier et al., 2006; Lawrie et al., 2006).

\section{METODOLOGIA PARA EL ANÁLISIS}

Es importante comprender que todas las organizaciones (y no está excluida la función mantenimiento) atraviesan sus propios procesos de madurez y que se trata de un proceso que debe preceder a excelencia. La madurez es la calidad o el estado de ser madura. Cuando se aplica el concepto de madurez a una organización operativa, se analiza el estado donde la organización se encuentra en relación a una condición ideal para conseguir sus objetivos (Andersen y Jessen, 2003).

La madurez de la organización para el mantenimiento significaría entonces que la organización está perfectamente condicionada para tratar sus proyectos. Pero la madurez dentro de la organización para el mantenimiento es explicada por la suma de la acción (habilidad de actuar y decidir), de la actitud (deseo de estar involucrado) y del conocimiento (una comprensión del impacto de la voluntad de hacer algo nuevo y de la acción).

Para hacer una evaluación de la madurez dentro de la función mantenimiento es preciso medir el estado en que la empresa se encuentra. Clarke y Garside (1997), proponen un modelo que combina cinco aspectos: compromiso, cultural, comunicación, herramientas y metodologías y gerenciamiento de conflictos.

Para el caso del mantenimiento, cada uno de estos aspectos significa lo siguiente:

a) Compromiso: incluye reconocer el cambio como una parte integral de la estrategia de la función, en concordancia con la estrategia de la organización, nivel de apropiación en toda la organización, más particularmente en el nivel de gerencia y formación de equipos de trabajo. También incluye la provisión de recursos adecuados para permitir que el proyecto funcione eficazmente y de la participación del personal en todos los niveles de la organización que tiene un relacionamiento con el mantenimiento en todas las fases del proyecto.

b) Cultural: se refiere principalmente a las personas como elemento del cambio, esto es, el asunto que involucra el comportamiento de las personas, de las percepciones y de las actitudes para todos los aspectos del cambio. Se refiere al nivel de la participación y del apoyo al cambio, no solamente del personal del mantenimiento, sino también incluye a producción, a administración de los recursos humanos y financieros y del personal de apoyo logístico y computacional.

c) Comunicación: considera todos los aspectos asociados con una comunicación interna y externa. Esto incluye la programación de los tiempos, métodos para una comunicación efectiva de los contenidos de los cambios. La finalidad principal es que todas las personas estén totalmente informadas sobre los cambios, los beneficios y como están comprometidas con la forma en que será hecho el mantenimiento en el presente momento. 
d) Herramientas y metodologías: aunque el interés predominante sea con el uso de la metodología de la gerencia de proyecto (equipos de proyecto y gerentes de proyecto), también se debe incluir el uso de medidas de comparación, de desempeño y de proceso. Cubre también el conocimiento subyacente requerido para asegurar que el cambio pueda ocurrir eficazmente y facilitar el uso de la entrada de conocimientos y del entrenamiento externo. Mantenimiento debe, en su área, liderar y propagar el uso de herramientas para tener un control y retroalimentación de sus acciones.

e) Gerenciamiento de los conflictos: se refiere a la preocupación de no interactuar negativamente con otros proyectos que ya están en aplicación dentro de la organización para tratar esos conflictos. Controlar el equilibrio entre las operaciones normales y los otros cambios que pueden acontecer en la organización y que ninguna de las mejorías hechas resulta en el desarrollo de uno de los factores claves de éxito a cuesta de otros. Debe haber un equilibrio entre los distintos factores que promueven el éxito de la producción.

Una organización puede ser madura en gestión y no ser excelente. La definición de excelencia va más allá de la definición de madurez. Cuando las organizaciones desarrollan sistemas y procesos maduros, surgen dos beneficios adicionales: primero, el trabajo es ejecutado con un mínimo de cambios de escenario y segundo, los procesos son definidos de manera de causar el mínimo de problemas para el negocio principal de la empresa (Kerzner, 2000).

Los estados de la madurez imponen condiciones distintas para la gestión ya que el escenario no puede ser el mismo para todas ellas. La fase más adversa (donde predomina el desorden) se caracteriza por el reconocimiento de una falencia del servicio y que se precisa cambiar para atender con éxito los objetivos de la empresa. En este estado, es muy difícil emprender un cambio de la gestión y se debe primero hacer un trabajo en todos los aspectos para que el personal empeñe todos sus esfuerzos para conseguir un mejor nivel. En esta etapa es muy importante el comprometimiento de la alta gerencia con el desarrollo de la función mantenimiento y la participación activa y efectiva del equipo de mantenimiento para desarrollar un proyecto de mejoramiento de la gestión.

En lo que concierne a la excelencia, Flynn et al. (1999) citan los fundamentos de Hayes y Wheelright mediante los cuales se manifiesta la excelencia o performance superior. En el caso del mantenimiento se pueden explicitar por las siguientes características: i) el personal del mantenimiento está altamente capacitado y sus conocimientos son transmitidos a las personas que hacen uso del equipamiento, ii) los administradores superiores son, en general, ingenieros y los demás tienen grados técnicos, iii) el mantenimiento siempre debe buscar el alineamiento de sus servicios y procedimientos para sustentar las necesidades de los equipamientos, iv) se desarrolló una cultura de confianza entre el personal de los varios departamentos, los trabajadores y administradores, v) existe una fuerte atención en la contribución al mejoramiento de la eficiencia global de la tecnología usada en la industria y v) la función mantenimiento se preocupa en avanzar en la tecnología de la información con el fin de evaluar con datos precisos, su desempeño y tener las bases para proponer e implementar acciones correctivas.

Cuando se aplica el concepto de madurez en una organización es para constatar el estado donde está en relación a los atributos que están siendo evaluados. La madurez de la función mantenimiento podría indicar que la organización definida para el mantenimiento está perfectamente condicionada para emprender todos sus proyectos de innovación, sean tecnológicos o de gestión integral de sus recursos. En el mundo real no se encuentra la madurez completa de una organización; nadie alcanzó el estado de máximo desarrollo y nadie lo hará (Andersen y Jessen, 2003). La razón es que el mundo de los negocios está siempre evolucionando, como también lo hacen las personas que componen la organización. Entonces tiene sentido hablar de grados de madurez y hacer esfuerzos para estar, en cada ciclo, mejor que en el anterior.

En la Fig. 1, se muestran los factores considerados en la metodología para estructurar el conjunto de preguntas, las cuales serán definidas aplicando la descomposición jerárquica propuesta por la metodología MCDA (Løken 2007), las cuales después de ser valoradas por los evaluadores, permitirán una visión del estado de la madurez de la función mantenimiento y de su entorno para cada un de los aspectos considerados. 


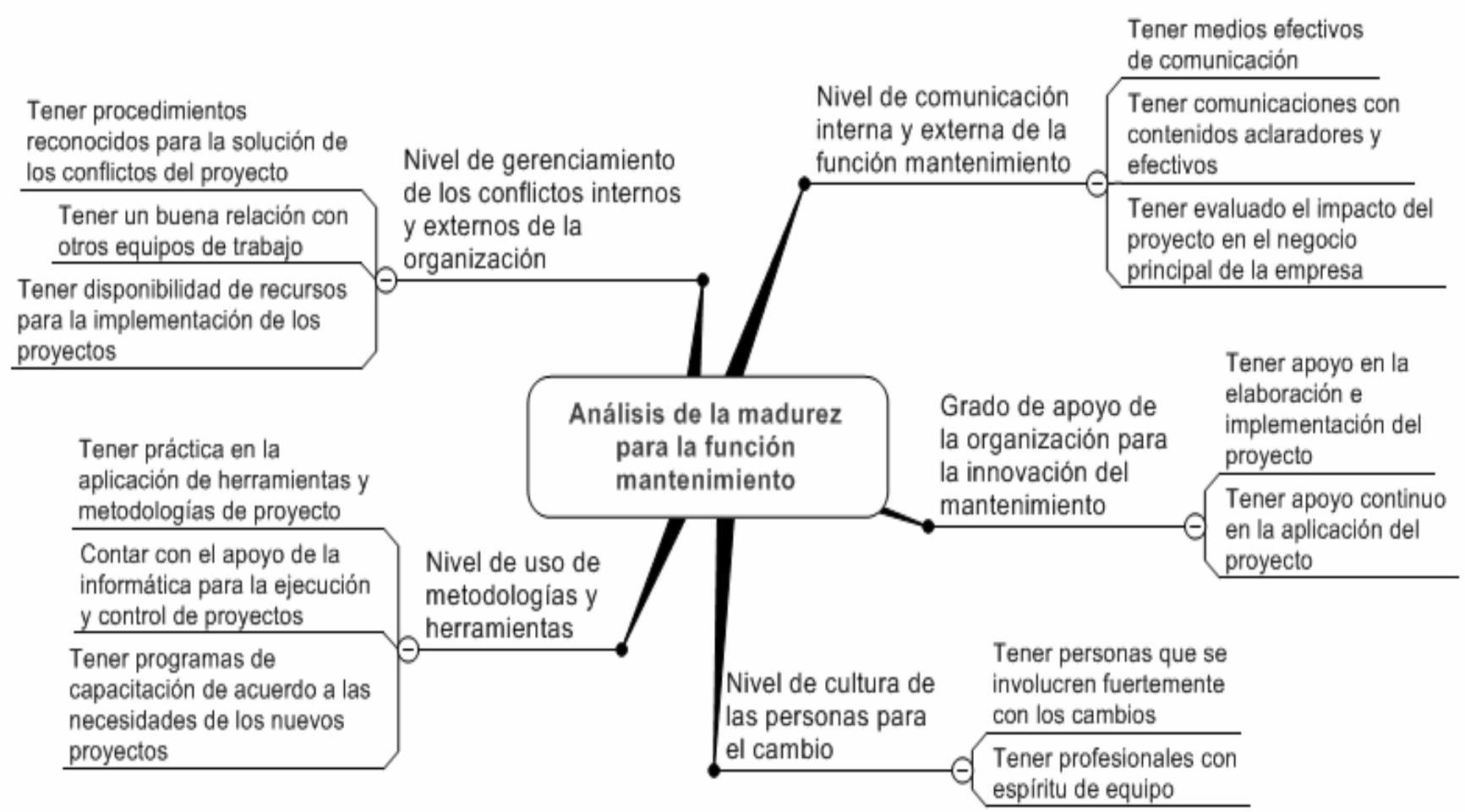

Fig. 1: Aspectos considerados para la evaluación de la madurez

La evaluación de cada respuesta es dada a partir de una escala de valores que van desde muy alto hasta sin interés o negativo, incluyendo la alternativa normal o esperada. Esta escala incluye el concepto de óptimo o máximo, que sería la referencia para tener seguridad que la implementación de un proyecto va a tener éxito.

La medición de la madurez es subjetiva y depende del juzgamiento del administrador de su entorno. La madurez es principalmente, la combinación del apoyo o comprometimiento que el entorno ofrece a la función mantenimiento más la capacidad o conocimiento que se tiene de las metodologías relacionadas con implementación de proyectos. Como hay voluntad de la organización de involucrarse en proyectos nuevos, hay cantidad y calidad de la información y capacidad de solucionar conflictos, la apreciación o evaluación del estado actual de madurez por parte del administrador, puede no estar muy lejana de la realidad.

Como todos los aspectos considerados no tienen la misma importancia, se deberá priorizar cada uno de estos antes de seguir con la aplicación de la metodología. Este punto es incluido en la metodología para considerarse y reflejar sobre el proceso de priorización que cada empresa da a cada aspecto de la madurez en cada proyecto de innovación en particular.

\section{ANÁLISIS DE LA MADUREZ DE LA ORGANIZACIÓN}

Este tópico es de alta relevancia y aquí el factor humano es clave para el desarrollo de aplicaciones innovadoras. Tiene influencia el grado previo de preparación y conocimiento sobre la innovación a implementar como también, la capacidad de transmitir el conocimiento (Nieto, 2004). La transición del "estado de evolución" a un "estado de revolución" requiere conocer en profundidad aspectos claves como la naturaleza del contexto externo de la empresa y las variables organizacionales tales como: visión organizacional, paradigmas conocidos del cambio, alineamiento estratégico, cultura organizacional, modos usados para el cambio, liderazgo dominante, estilos de administración, aprendizaje organizacional y las metas y resultados esperados (Hill y Collins, 2000).

Siguiendo las sugerencias de Clarke y Garside (1997) para un análisis de madurez, fue incluido en la metodología cinco aspectos: compromiso, cultural, comunicación, gestión de los conflictos, herramientas y metodologías, los cuales serán evaluados en base en los conceptos ya definidos. Las 
preguntas realizadas al administrador, (Fig. 2), son posteriormente agrupadas en la forma de diagrama radar (Fig. 3), para evidenciar mejor el estado actual en relación al campo de posibilidades para un mayor grado de madurez.

El informe que acompaña al análisis de madurez es similar para todos los aspectos considerados, y está constituido de tres partes, destacados en el ejemplo mostrado (Fig. 4 y 5). La primera parte (Fig. 4) enfoca los aspectos más globales considerados en el análisis y se refieren al tipo de apoyo que se tendrá durante la implementación de la innovación y el apoyo posterior para sustentar y ampliar la aplicación de la concepción del mantenimiento. La segunda parte del informe se refiere al proceso de análisis específicos para los aspectos de la madurez "tener apoyo continuo en el desarrollo" y para "tener apoyo en la implementación y elaboración de proyectos". Por ejemplo, los valores obtenidos en la Fig. 4, están próximos de 2,5, lo que en la escala ponderada indica estar entre "dificultad y difícil y normal o bueno" (Fig.2). Tiene la función de explicar el porqué del nivel de los conceptos globales y de esta forma conocer las causas del nivel de madurez actual, para mejor identificar las herramientas que auxilian en la evolución del nivel de madurez.

La tercera parte entrega un análisis de los aspectos puntuales, que son complementarios, relacionando los que deberían ser compatibles (Fig. 5). Esta información es útil para detectar aspectos que pueden afectar la implementación del proyecto. Por ejemplo, la relación que existe entre el interés en participar, por parte de profesionales de otras áreas y el apoyo que otorgan los dirigentes de esas áreas. Si el superior no apoya el proyecto, el profesional subordinado ciertamente no contribuirá de forma eficiente al proyecto.

\section{Evaluar el grado de apoyo continuo de la organización para innovación del mantenimiento}

\section{Proceso de diagnóstico}

Ingresar el valor 1 en las celdas verde que mejor describen su situacion actual

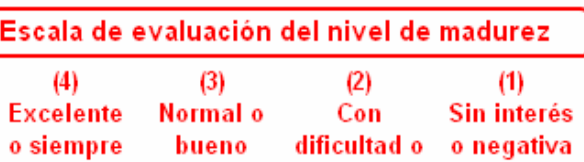
difici

Tener apoyo continuo en la aplicación del projecto de mantenimiento

P1.1 ¿ Los operadores de la función mantenimiento tienen la posibilidad de asistir a las reuniones gerenciales, exponer los objetivos de los proyectos de innovación y recibir sugerencias?

P1.2 ¿ La función mantenimiento elabora informes completos de sus proyectos emprendidos a interior de la organización con relación a los aportes entregados?

P1.3 ¿Se recibe de la gerencia y de otros departamentos comentarios y aportes para el proyecto de innovación del mantenimiento?

P1.4 iCuando se llama a una reunión considerada importante para el análisis de los avances de un proyecto, siempre tiene la cooperación de otros sectores de la organización?

P1.5 ¿Cuando se constituye un equipo de trabajo para un proyecto, la asistencia de los profesionales llamados a participar es siempre continua y regular?

P1.6 ¿Cada participante del proyecto de innovación es informado y valoriza la importancia de su participación?

P1.7 Le incentiva a los miembros del equipo de proyecto y se tiene reconocimiento constante para así traer mejorías para el proyecto de innovación?

P1.8 ¿En los proyectos de innovación elaborados por mantenimiento, se identifican bien las áreas críticas y se hace un análisis económico del proyecto?

P1.9 ¿En los proyectos de innovación elaborados por mantenimiento, se identifican bien los beneficios y ganancias que obtendrian otras áreas de la organización?

P1.10 ¿Es divulgado de manera formalizada (murales, periódicos) los aportes de las personas al proyecto y al avance de la innovación?

P1.11 ¿La alta administración de la organización ve al mantenimiento como un diferencial competitivo para la empresa?

P1.12 ¿Existe en la organización la estructura para crear un banco de datos con proyectos que integran diferentes áreas de la empresa?

P1.13 ¿Se tiene en la organización procedimientos, criterios e información para priorizar los proyectos?

Tener apoyo en la implementación y elaboración del proyecto

P1.14: Hay condiciones favorables de carga de trabajo, para los mantenedores y otros profesionales tal que permita participar en grupos de estudo de proyectos de innovación? P1.15: Se tiene respuesta positiva de los profesionales relacionados con el mantenimiento para integrarse a grupos de estudio para proyectos de innovación?

P1.16: Existe una sinergia entre los líderes de proyectos para apoyarse, compartir recursos e información cuando los provectos son converaentes?

\begin{tabular}{|l|l|l|l|}
\hline & 1 & 1 & \\
\hline & & 1 & \\
\hline & & 1 & \\
\hline & 1 & & \\
\hline & 1 & & \\
\hline & 1 & & \\
\hline & 1 & & \\
\hline & 1 & & \\
\hline & & 1 & \\
\hline
\end{tabular}

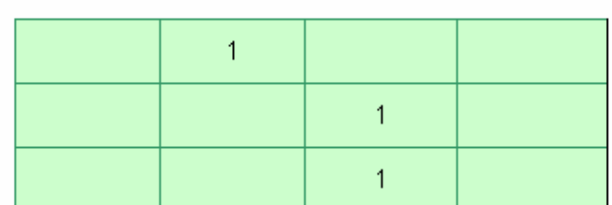

Fig. 2: Parte de la captura de datos para la evaluación del aspecto grado de apoyo de la organización 
El resultado final del análisis de la madurez se obtiene mediante la media ponderada de los cinco factores considerados en el análisis. El administrador define las ponderaciones de cada factor para que se refleje su importancia en relación al futuro de la aplicación de la concepción del mantenimiento. Así, si el administrador estima que los conflictos no son relevantes durante el proceso de implementación, porque existen recursos suficientes para todos los proyectos y los integrantes de otros equipos de proyecto o áreas de la empresa no van a ser afectados por la implementación del proyecto de innovación, se atribuye a este aspecto un valor bajo para la evaluación final (Fig. 6).

Además, si la evaluación de algún punto fuese negativa, se dan orientaciones de posibles acciones que podrían ser implementadas para minimizar el efecto no deseado, además de un modelo de jerarquización de estas actividades basado principalmente en el nivel de madurez de cada variable analizada (Fig. 7). Mejorar la madurez trae como consecuencia dos ventajas: reducir la variabilidad inherente al proceso y mejorar la eficiencia media del proceso (Cooke-Davies y Arzymanowc, 2003).

En este punto de la aplicación de la metodología el administrador debe responde la siguiente pregunta: ¿el nivel de madurez de la organización es el adecuado para enfrentar el proceso de innovación? La respuesta debe ser entregada basada en el conocimiento y en la experiencia que el administrador tiene sobre su entorno. Se puede dar el caso que el nivel de madurez está muy próximo a lo que el administrador considera como lo recomendado o esperado para la aplicación de la innovación en el mantenimiento, de ser así, puede poner en práctica en paralelo las mejorías sugeridas en la metodología en conjunto con la implementación de la concepción, si el tiempo que tiene definido para responder a los requisitos de la empresa es corto.

Informe para el grado de apoyo continuo de la organización para innovación del mantenimiento

Cada aspecto considerado para la evaluación de la madurez tiene el siguiente nivel:

Evaluar el grado de apoyo continuo de la organización para innovación del mantenimiento 2.5

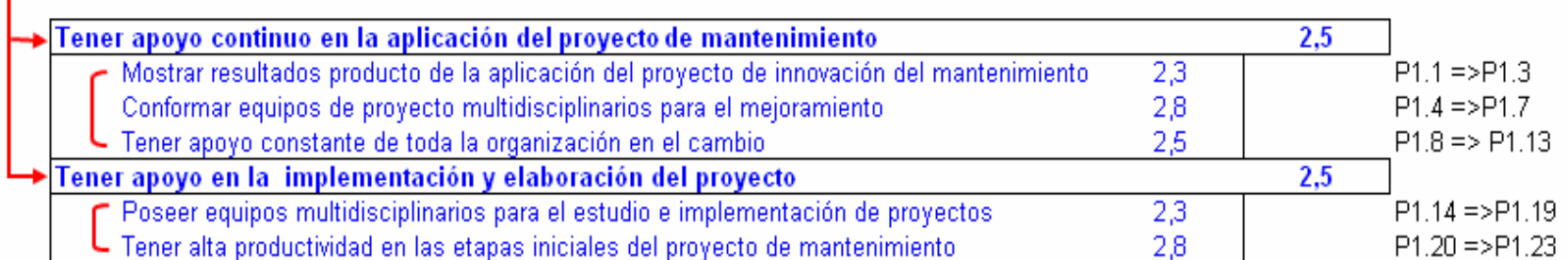

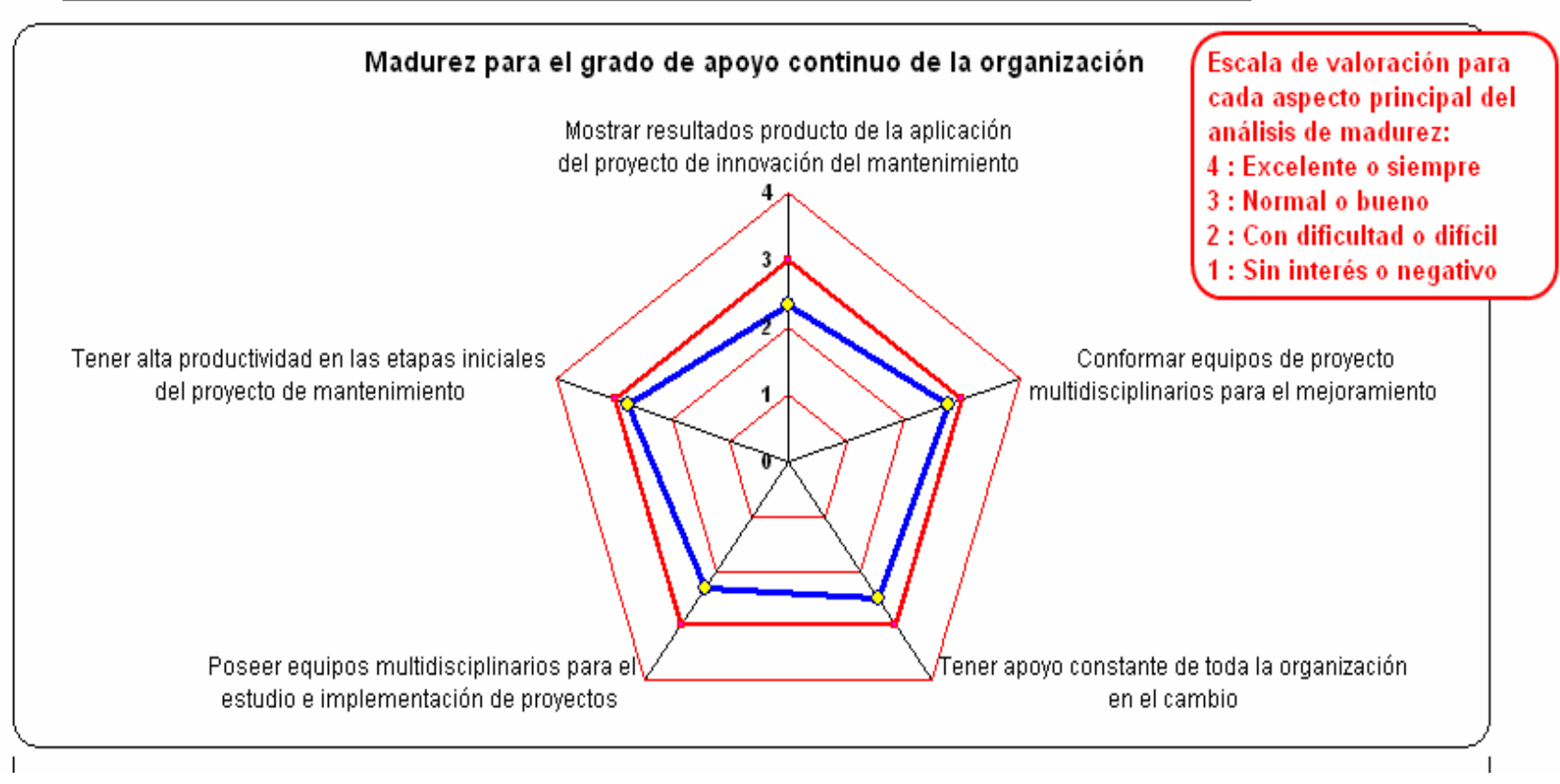

Fig. 3: Cuadro con los conceptos para el aspecto del apoyo continúo. 


\section{JERARQUIZACIÓN DE LAS ACTIVIDADES}

Un estudio sobre la madurez tiene entre otras finalidades ayudar a la organización a reconocer cuándo y por que debe mejorar, como también como obtener una visión de la propia realidad y, así, conocer cuales acciones necesita emprender para conseguir alcanzar sus objetivos (Duffy, 2001). De esta forma, se asegura de proveer los medios adecuados para mejorar el desempeño de cada integrante y de la organización como un todo (Harrison, 1995).

El foco central del modelo está en procurar cual acción de mejoramiento, dentro de todas las sugeridas, provoca el mayor impacto a través de todos los aspectos considerados en el estudio de la madurez y, de esta forma ser más efectivo en la aplicación de las acciones seleccionadas.

El criterio de ordenamiento (Ec. 1) - Cri- (Orden de las prioridades para la implementación) es el producto ponderado entre el grado de madurez de cada aspecto $i$-GMi - (Nivel actual de la madurez) determinado en la aplicación de la metodología y el número de impactos de cada acción $j$ en el conjunto de aspectos $i$ - imp $p_{i, j}$ - (Número de impactos). Detalles en Fig. 7.

$$
\begin{aligned}
& \text { Para cada acción } j \\
& \text { Cr }_{i}=\sum_{i=1}^{n} \operatorname{imp}_{i, j} \times G M_{i} \\
& i=1,2, \ldots, n \text { aspectos }
\end{aligned}
$$

Para el análisis relacionado con el grado de apoyo continuo de la organización para innovación del mantenimiento, se pueden observar las siguientes características:

\begin{tabular}{|c|c|}
\hline Tener apoyo continuo en la aplicación del proyecto de mantenimiento & Nivel \\
\hline \multicolumn{2}{|c|}{$\begin{array}{l}\text { Mostrar resultados obtenidos por el desarrollo de proyectos: } 2,3 \\
\text { Itado es conocido sólo a nivel de departamentos y los niveles superiores no tienen conocimiento de todos los } \\
\text { ios del proyecto }\end{array}$} \\
\hline \multicolumn{2}{|c|}{$\begin{array}{l}\text { Conformar equipos para el mejoramiento de los proyectos: } \\
\text { Los equipos de trabajo son formados normalmente pero en general no llegan todos los convocados y la probabilidad de } \\
\text { ausencias o rotación es alta }\end{array}$} \\
\hline \multicolumn{2}{|c|}{$\begin{array}{l}\text { Tener apoyo constante de toda la organización en el cambio: } \\
\text { El apoyo es normal y si se precisar de apoyo este es entregado pero no de inmediato. La atención de la gerencia está } \\
\text { vuelta hacia proyectos más rentables }\end{array}$} \\
\hline \multicolumn{2}{|c|}{ Aspectos específicos } \\
\hline Tener apoyo en la implementación y elaboración del proyecto & Nivel \\
\hline $\begin{array}{l}\text { La conformación de equipos para la implementación de proyectos: } \\
\text { Los equipos de trabajo son formados normalmente pero en general no llegan todos los convoca } \\
\text { ausencias o rotación es alta. }\end{array}$ & $\begin{array}{l}2,3 \\
\text { y la probabilidad de }\end{array}$ \\
\hline $\begin{array}{l}\text { La productividad del proyecto en las etapas iniciales: } \\
\text { El proyecto puede tener aspectos que no serán bien desarrollados y puede tener una probabilid } \\
\text { en su implementación }\end{array}$ & $\begin{array}{l}2,8 \\
\text { alta de dar problema }\end{array}$ \\
\hline
\end{tabular}

\begin{tabular}{|c|c|}
\hline \multicolumn{1}{|c|}{ Aspectos globales } & Nivel \\
\hline Tener apoyo continuo en la aplicación del proyecto de mantenimiento : & 2,5 \\
Tener apoyo inconstante por parte de la gerencia o de las áreas donde el proyecto tiene influencia, o sea, el proyecto \\
tiene un seguimiento complicado \\
Tener apoyo en la implementación y elaboración del proyecto: & 2,5 \\
Tener apoyo inconstante o dificil por parte de la gerencia, o sea, para que el proyecto tenga exito debe tener una \\
preocupación extra del lider
\end{tabular}

Aspectos específicos

Fig. 4: Informe para el análisis de madurez referida al apoyo de la organización

Se ordenan de menor a mayor, priorizando de esta manera a las acciones que tienen el mayor número de impacto sobre los aspectos con más bajo nivel de madurez. 


\section{Análisis complementario}

Para relaciones de aspectos específicos se tiene la siguiente evaluación

\begin{tabular}{|l|l|l|}
\hline $\begin{array}{l}\text { La gerencia tiene herramientas para priorizar los proyectos, pero estas no son bien elaboradas ni ejecutadas } \\
2\end{array}$ & Alerta \\
\hline $\begin{array}{l}\text { Las condiciones de carga de trabajo internas permiten participar en grupos de estudio de proyectos de mantención pero } \\
\text { los líderes de otros proyectos no apoyan a otros que son convergentes }\end{array}$ & Alerta \\
\hline $\begin{array}{l}\text { En los consejos superiores se tiene la oportunidad de exponer los proyectos, pero no recibe información que pueda ser útil } \\
\text { para la mejoría del proyecto }\end{array}$ & Alerta \\
\hline $\begin{array}{l}\text { No tiene una asistencia a las reuniones muy alta o con aquellas personas claves para o proyecto, pero en el momento de } \\
\text { iniciarlo llegan todos los profesionales importantes }\end{array}$ & Alerta \\
\hline $\begin{array}{l}\text { Incentivar a los miembros del equipo de trabajo para traer mejorías y tener un medio eficaz para divulgar los aportes de las } \\
\text { personas están correlacionados de forma positiva }\end{array}$ & Positivo \\
\hline $\begin{array}{l}\text { Los proyectos no son desarrollados con todos los aspectos bien identificados y evaluados, pero se consigue la participación } \\
\text { de profesionales del staff superior. Alerta esto no siempre funciona }\end{array}$ & Alerta \\
\hline $\begin{array}{l}\text { Los proyectos no son desarrollados con todos los aspectos bien identificados y evaluados, pero se consigue tener una } \\
\text { aprobación por los involucrados. Puede indicar que su proyecto fue analizado superficialmente }\end{array}$ & Alerta \\
\hline $\begin{array}{l}\text { El proyecto no está bien definido en lo relativo a los problemas que va a solucionar pero está con todos los beneficios bien } \\
\text { identificados. Esto podría dar una razón costo-beneficio mayor que } 1\end{array}$ & Alerta \\
\hline $\begin{array}{l}\text { Tener una respuesta positiva de los profesionales y el esfuerzo que la empresa destina para combinar proyectos } \\
\text { congruentes están correlacionados de forma negativa }\end{array}$ & Negativo \\
\hline $\begin{array}{l}\text { Los proyectos son aprobados por la gerencia pero los recursos no están disponibles en el momento del inicio. Verifique para } \\
\text { el caso de proyectos similares y estime el tempo de espera, mientras perfeccione su proyecto. }\end{array}$ & Alerta \\
\hline
\end{tabular}

Fig. 5: Informe con aspectos complementarios para el apoyo de la organización

\section{Informe para el análisis de la madurez para la función mantenimiento}

\begin{tabular}{|lr|}
\hline Grado de apoyo continuo de la organización para la innovación del mantenimiento: & 2,5 \\
Nivel de cultura de las personas para enfrentar cambios: & 2,5 \\
Nivel de comunicación interna y externa de la función mantenimiento: & 2,8 \\
Nivel de uso de metodologías y herramientas de gestión: & 2,6 \\
Nivel de gerenciamiento de los conflcitos internos y extemos de la empresa: & 2,7 \\
\hline
\end{tabular}

\section{Proceso de diagnóstico}

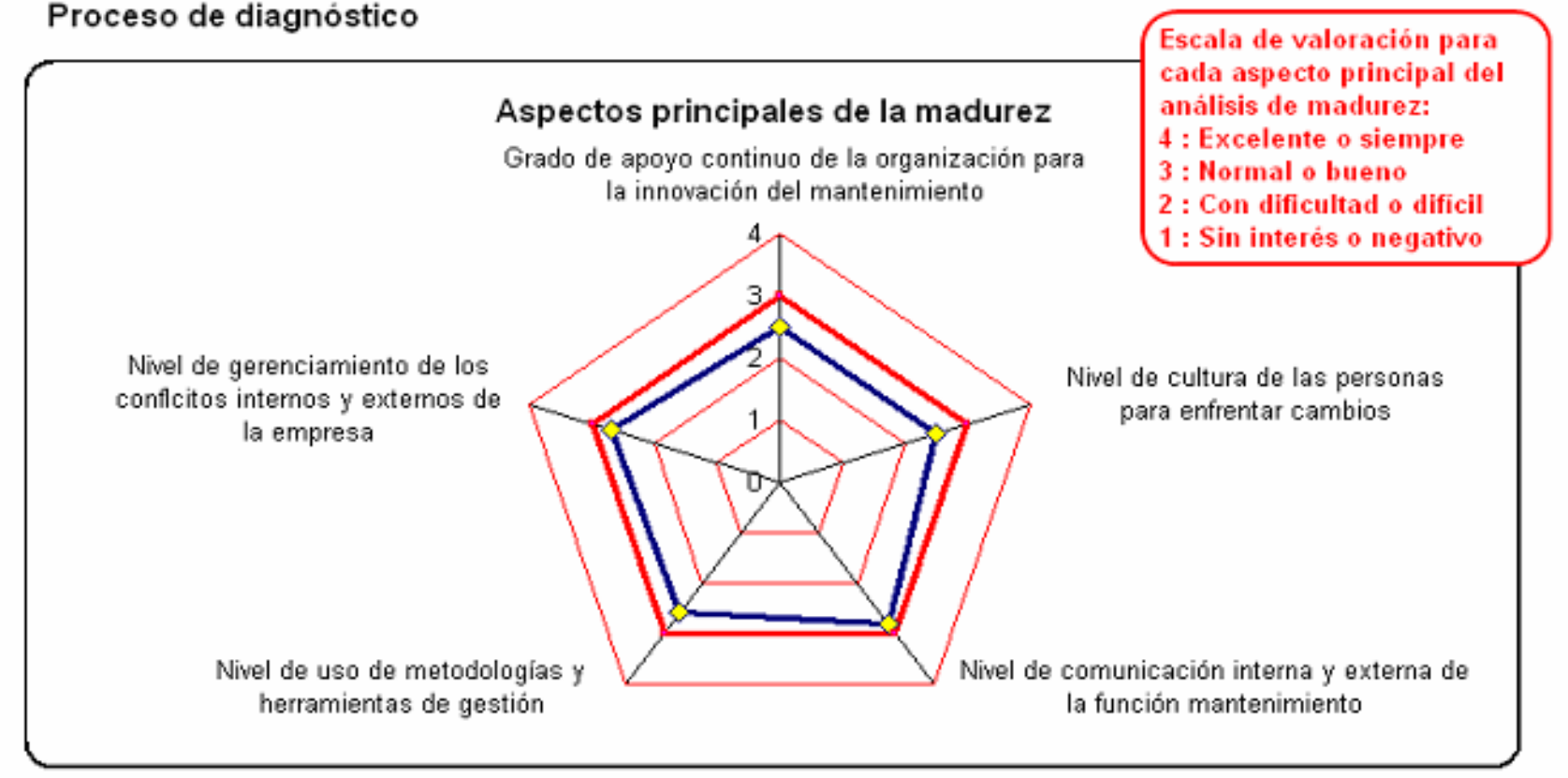

Considerando las ponderaciones de cada aspecto, el resultado global de la madurez de la organización da un valor de: 2,6 que puede ser considerado como: nivel normal pero inconstante donde se precisa persistencia del equipo de trabajo en especial del lider.

Fig. 6: Cuadro resumen con todos los conceptos medidos de la madurez de la organización 
El modelo mostrado en la Fig. 7, como ejemplo, considera solamente los aspectos relacionados con el apoyo de la organización en la implementación de proyectos de innovación para la gestión del mantenimiento y la cultura de la organización en relación a los cambios. La definición de las acciones de mejoramiento, cuando se aplica la metodología, en principio está enfocada para resolver el problema puntual de cada variable de la madurez, pero en el modelo de jerarquización de estas acciones ya se tiene la visión holística del problema. De esta forma, se define el concepto de impacto de la acción.

Se puede dar la situación que dos o más factores tengan el mismo valor para el criterio de ordenamiento, pero la condición que dio origen a estos valores puede ser muy diferente para cada uno de ellos. Se debe prestar mayor atención cuando suceda esta situación ya que una decisión basada en un análisis sin profundizar en sus causas puede llevar a resultados errados.

\begin{tabular}{|c|c|c|c|c|c|c|c|c|c|c|c|c|}
\hline & $\mathrm{Nec}$. & & Nec. & Nec. & & Nec. & Nec. & Nec. & Nec. & Nec. & & \\
\hline 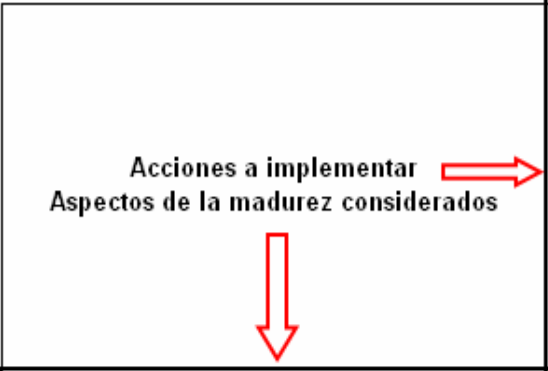 & 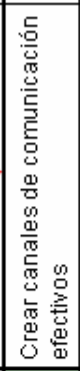 & 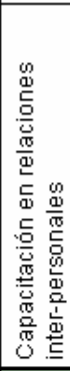 & 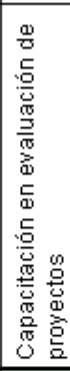 & 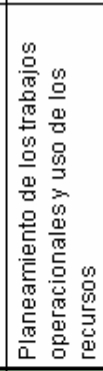 & 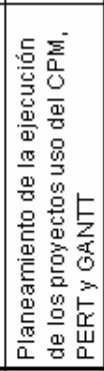 & 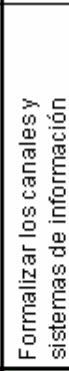 & 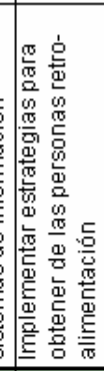 & 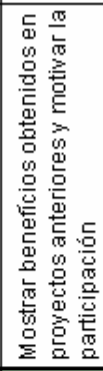 & 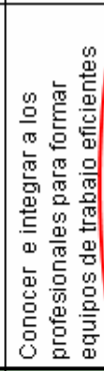 & 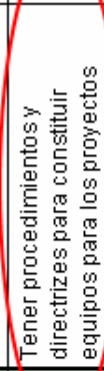 & 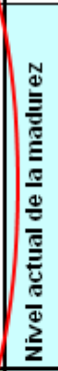 & \\
\hline $\begin{array}{l}\text { Mostrar resultados obtenidos por el desarrollo de } \\
\text { projectos de innovación del mantenimiento }\end{array}$ & 1 & & & & 1 & 1 & & 1 & & & 2,3 & \\
\hline $\begin{array}{l}\text { Conformar equipos multidisciplinarios para el } \\
\text { mejoramiento de los proyectos }\end{array}$ & & 1 & & & & & & 1 & & & 2,8 & \\
\hline $\begin{array}{l}\text { Tener apoyo constante de toda la organización en el } \\
\text { cambio }\end{array}$ & 1 & & & & & 1 & & 1 & & & 2,5 & $\begin{array}{l}\text { Introducir el } \\
\text { valor } 1 \text { cuando }\end{array}$ \\
\hline $\begin{array}{l}\text { Poseer equipos multidisciplinarios para estudio e } \\
\text { implementación de proyectos }\end{array}$ & & 1 & & 1 & & & & & 1 & 1 & 2,3 & \\
\hline $\begin{array}{l}\text { Tener alta productividad en las etapas iniciales del } \\
\text { proyecto de mantenimiento }\end{array}$ & 1 & & 1 & 1 & 1 & & 1 & & 1 & & 2,8 & $\begin{array}{l}\text { sobre la variable } \\
\text { de madurez }\end{array}$ \\
\hline $\begin{array}{l}\text { Tener alto nivel de apoyo para la implementación de } \\
\text { los cambios }\end{array}$ & & 1 & & & & 1 & & 1 & & & 2,3 & \\
\hline $\begin{array}{l}\text { Obtener un reconocimiento positivo para los } \\
\text { cambio }\end{array}$ & 1 & & & & & & & 1 & & & 2,5 & \\
\hline $\begin{array}{l}\text { Tener entre las personas una cultura para los } \\
\text { cambios }\end{array}$ & & & & & & & 1 & 1 & & & 2,4 & \\
\hline $\begin{array}{l}\text { Tener equipos de personas con alta eficiencia en el } \\
\text { trabajo }\end{array}$ & & 1 & 1 & & & & 1 & & 1 & & 2,7 & \\
\hline $\begin{array}{l}\text { Constituir equipos de profesionales homogeneos } \\
\text { de trabajo }\end{array}$ & & 1 & & & & & & & 1 & 1 & 2,5 & \\
\hline Número de impactos & 4 & 5 & 2 & 2 & 2 & 3 & 3 & 6 & 4 & 3 & & \\
\hline Orden de las prioridades para la implementación & 5 & 4 & 10 & 6 & 6 & 1 & 9 & 2 & 8 & 3 & & \\
\hline
\end{tabular}

Fig. 7: Matriz de prioridades para acciones relacionadas con la madurez de la organización

\section{CONCLUSIONES}

Este modelo fue desarrollado para facilitar el proceso de innovación en la gestión del mantenimiento con la finalidad de poseer una herramienta para evaluar el entorno en el cual la función está inserida. En su aplicación se pudo constatar que el concepto de madurez es vagamente conocido por la mayoría de los administradores del mantenimiento, lo cual explica en gran parte los problemas en la eficiencia de la gestión y que redundan de forma adversa al momento de querer introducir innovaciones en su proceso. Es así que durante la aplicación y validación del modelo se consumió un tiempo apreciable, porque fue la oportunidad que muchos de los encargados del mantenimiento en las empresas consultadas tuvieron para cuestionar su gestión en relación a cada aspecto incluido en el modelo, como también para pensar en planes y estrategias para mejorar la relación de trabajo con el resto de la organización. 
Para evaluar cada aspecto las preguntas se formularon de tal forma de circunscribirse a la esencia de cada aspecto incluido en el modelo, porque cuando se alcanza un grado aceptable de madurez existe una relación de dependencia entre ellos. Si hay una cultura para los cambios no es difícil tener un compromiso para apoyar la innovación y si hay compromiso también hay voluntad para evitar los conflictos, lo cual revela que alcanzar la madurez es un proceso que requiere la cooperación de muchos estamentos dentro de la organización.

\section{REFERENCIAS}

Abrunhosa A. y P. Moura e Sá; Are TQM principles supporting innovation in the Portuguese footwear industry? Technovation: 28, 208-221 (2008).

Andersen E. y S. A. Jessen; Project maturity in organizations. International Journal of Project Management 21, 457-461 (2003).

Clarke A. y J. Garside; The development of a best practice model for change management. European Management Journal 15 (5) (1997).

Cooke-Davies T. y A. Arzymanowc; The maturity of project management in different industries: An investigation into variations between project management models. International Journal of Project Management 21. 471- 478 (2003).

Duffy J.; Maturity models blueprints for e-volution. Strategy \& Leadership 29, 19-26 (2001).

Flynn B., R. Schroeder y J. Flynn; World class manufacturing: an investigation of Hayes and Wheelwright's Foundation. Journal of Operations Management 17. 249-260 (1999).

Garengo P., S. Nudurupati y U. Bititci; Understanding the relationship between PMS and MIS in SMES: An organizational life cycle perspective. Computers in Industry 58, 677- 686 (2007).

Harrison E.; Strategic planning maturities. Management Decision 33 (2), 48-55 (1995).

Hill F. y L. Collins; A descriptive and analytical model of organizational transformation. Int. Journal of Quality \& Reliability Management 17 (9), 966-983 (2000).

Kerzner H. Gestão de projetos, as melhores práticas. Editora Bookman, (2000).

Lawrie M., D. Parker y P. Hudson; Investigating employee perceptions of a framework of safety culture maturity. Safety Science 44, 259-276 (2006).

Løken E.; Use of multicriteria decision analysis methods for energy planning problems. Renewable and Sustainable Energy Reviews 11 1584-1595 (2007)

Maier A., C. Eckert y P. Clarkson; Identifying requirements for communication support: A maturity grid-inspired approach. Expert Systems with Applications 31, 663- 672 (2006).

Nieto M. Basic propositions for the study of the technological innovation process in the firm. European Journal of Innnovation Management. 7(4), 314-324 (2004).

Xirogiannis G. y M. Glykas; Intelligent modeling of e-business maturity. Expert Systems with Applications 32, 687-702 (2007). 